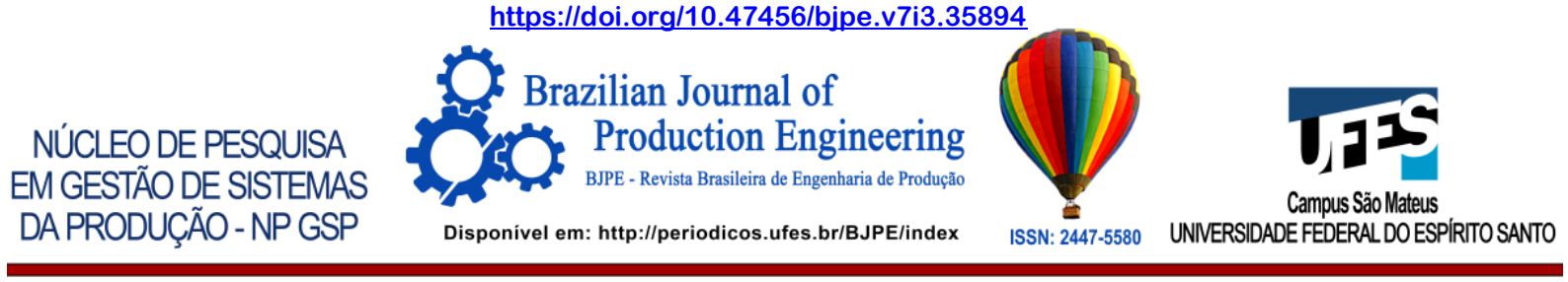

ARTIGO ORIGINAL

OPEN ACCESS

\title{
REDUÇÃO DA VISCOSIDADE DO BIODIESEL DE Ricinus communis L. (MAMONA) POR ACETILAÇÃO QUÍMICA
}

\section{REDUCTION OF THE VISCOSITY OF BIODIESEL FROM Ricinus communis L. (CASTOR BEAN) BY CHEMICAL ACETYLATION}

\section{$\underline{\text { Carmem Cícera Maria da Silva }}{ }^{1 *}$, Leila Cristina Konradt Moraes ${ }^{2}$, José Ribeiro dos $\underline{\text { Santos Júnior }}^{3}, \&$ Thiago Luis Aguayo de Castro ${ }^{4}$

\footnotetext{
${ }^{1}$ Universidade Federal do Espírito Santo, UFES, Brasil. ${ }^{24}$ Universidade Estadual de Mato Grosso do Sul, UEMS, Brasil. ${ }^{3}$ Universidade Federal do Piauí, UFPI, Brasil.

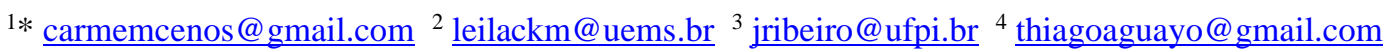

\section{ARTIGO INFO.}

Recebido em: 29.06.2021

Aprovado em: 09.09.2021

Disponibilizado em: 16.09.2021

Palavras-chave:

Derivatização; RMN; química.

KEYWORDS:

Derivatization; RMN; chemistry.

*Autor Correspondente: Silva, C. C. M, da.

\section{RESUMO}

O óleo da semente de mamona (Ricinus communis L.) é utilizado para a produção de biodiesel devido a sua resistência e ao fato de não competir com a produção alimentícia, contudo a elevada concentração de ácido ricinoleico em sua composição química conferem ao óleo de $R$. communis algumas características atípicas, como a elevada viscosidade. Portanto, esta pesquisa objetivou analisar a reação de acetilação dos ésteres ricinoleicos como alternativa de reduzir a viscosidade do biodiesel de $R$. communis. O óleo das sementes de $R$. communis foi caracterizado e transesterificado por via metílica avaliando o tempo reacional (30 minutos e 60 minutos), posteriormente foi realizado a acetilação utilizando o anidrido acético como reagente e piridina como catalisador. Realizou-se ensaios físico-químicos do óleo e biodiesel e RMN de ${ }^{1} \mathrm{He} \mathrm{e}^{13} \mathrm{C}$ do óleo e biodiesel acetilado. As propriedades físicoquímicas estavam dentro das especificações, com exceção de densidade e viscosidade. A análise por $\mathrm{RMN}$ de ${ }^{1} \mathrm{H}$ e ${ }^{13} \mathrm{C}$ demonstraram a presença majoritária de ácido ricinoleico no óleo de $R$. communis e comprovaram a eficiência da reação de acetilação. A acetilação reduziu a viscosidade em
$30,45 \%$ pelo método A e $32,66 \%$ no B. A acetilação química se apresenta como uma alternativa para a redução da viscosidade do biodiesel de $R$. communis.

\begin{abstract}
Castor seed oil (Ricinus communis L.) is used for biodiesel production due to its resistance and the fact that it does not compete with food production, however a high concentration of ricinoleic acid in its chemical composition gives the oil of $R$. communis some atypical characteristics, such as high viscosity. Therefore, this research aimed to analyze an acetylation reaction of ricinoleic esters as an alternative to reduce the viscosity of $R$. communis biodiesel. The oil from the seeds and R. communis was characterized and transesterified by two methods ( $A$ with 30 minutes and $B$ with 60 minutes), then acetylation was performed using acetic anhydride as a reagent and pyridine as a catalyst. The physicochemical tests of the oil and biodiesel and ${ }^{1} \mathrm{H}$ and ${ }^{13} \mathrm{C} \mathrm{NMR}$ of the oil and acetylated biodiesel were carried out. The physical-chemical properties of the specifications, with the exception of density and viscosity. An analysis by ${ }^{1} \mathrm{H}$ and ${ }^{13} \mathrm{C} N \mathrm{NM}$ showed a major presence of ricinoleic acid in the oil of $R$. communis and proved the efficiency of the acetylation reaction. Acetylation reduced viscosity by $30.45 \%$ in method $A$ and $32.66 \%$ in method B. Chemical acetylation is an alternative for reducing the viscosity of R. communis biodiesel.
\end{abstract}


Citação (APA): Silva, C. C. M. da, Moraes, L. C. K., Santos, J. R. dos, Jr, \& Castro, T. L. A. de (2021). Redução da viscosidade do biodiesel de Ricinus Communis L. (mamona) por acetilação química. Brazilian Journal of Production Engineering, 7(3), 182-198.

\section{INTRODUÇÃo}

A Ricinus communis L., conhecida popularmente como mamona, é uma pequena árvore que pode crescer até 6 metros presente em diversos países como o África do Sul, Brasil, Índia e Rússia (Rana, Dhamija, Prashar, \& Sharma, 2012). Esta espécie se adapta bem a temperaturas entre $20^{\circ} \mathrm{C}$ e $30^{\circ} \mathrm{C}$ e é capaz de resistir a períodos de seca (Assunção, Valdevino \& Rodrigues, 2020). Sua boa adaptação nestes países se dá pela baixa necessidade de água que se adequa muito bem a regiões semiáridas (Cangemi, Santos, \& Neto, 2010). Este cultivo pode ser considerado energeticamente eficiente, além de uma rica fonte de matéria orgânica (Chechetto, Siqueira, \& Gamero, 2010).

A semente de R. communis possui um alto teor lipídico, entre $40 \%$ e $55 \%$ (Keera, El Sabagh \& Taman, 2018). Este óleo possui uma enorme versatilidade química dentro do ramo industrial, podendo ser empregado em vários processos industriais: fabricação de tintas, lubrificantes, cosméticos, drogas farmacêuticas, corantes, desinfetantes, germicidas, colas e fungicidas (Lima et al., 2011, Azevedo \& Lima, 2001).

As diversas aplicações do $R$. communis estão associadas a composição química do óleo é atípica se comparada com a maioria dos óleos vegetais (Azevedo \& Lima, 2001). Segundo os mesmos autores, este óleo apresenta uma elevada concentração de ácido ricinoleico, que possui uma Hidroxila no Carbono 12 da estrutura graxa, conferindo algumas de suas propriedades intrínsecas, como a elevada viscosidade, sua miscibilidade em álcool e a reatividade diferente dos demais ácidos graxos. Estas características ocorrem por causa da capacidade da Hidroxila do Carbono 12 de realizar ligações de hidrogênio (Assunção et al., 2020).

Um dos usos industrias para os óleos é a produção de biodiesel, um biocombustível que possui implicações socioeconômicas e contribui efetivamente a reduzir o impacto ambiental associado ao uso de combustíveis fósseis (Barros, Purificação, Campanha, Silva, \& Santos, 2020). Suas características são semelhantes ao diesel, apresentando segurança ao ser estocado, contendo baixo teor de enxofre, ausência de toxicidade, sendo biodegradável e possuindo menor emissão de poluentes em relação aos combustíveis fósseis (Singh et al., 2020; Sia, Kansedo, Tan, \& Lee, 2020). Este biocombustível é formado por ésteres de alquila proveniente da transesterificação de ácidos carboxílicos de cadeia longa, oriundo de origem vegetal, animal (Costa, Sitoe, Santos, \& Neto, 2020; Silva, Carvalho \& Fonseca, 2021) ou de microrganismos (Oliveira, D'alessandro, Antoniosi Filho, Lopes, \& Derner, 2021). Recentemente, pesquisas vem buscando fontes de lipídios que não compitam com fontes vegetais que podem ser destinadas a alimentação humana (Borges et al., 2016; Konradt-Moraes, Oliveira Junior, Cardoso, \& Vieira, 2019; Postaue, Konradt-Moraes, \& Asmus, 2020; Olivera Junior, KonradtMoraes, Castro, \& Santos, 2021).

Devido ao alto teor lipídico e resistência no cultivo, o uso do óleo das sementes de $R$. communis para produção de biodiesel se popularizou (Rizzardo, Milfont, Silva, \& Freitas, 2012). Diversos estudos tem sido realizados para otimizar a produção (Ozcanli, Akar, Calik, \& Serin, 2017; Das, Sarkar, Datta, \& Santra, 2018; Keera et al., 2018; Elango et al., 2019), contudo, o biodiesel produzido possui uma viscosidade elevada em relação a outras oleaginosas, devido à presença de ácido ricinoleico em sua composição (Cangemi et al., 2010; Elango et al., 2019; Osorio- 
Citação (APA): Silva, C. C. M. da, Moraes, L. C. K., Santos, J. R. dos, Jr, \& Castro, T. L. A. de (2021). Redução da viscosidade do biodiesel de Ricinus Communis L. (mamona) por acetilação química. Brazilian Journal of Production Engineering, 7(3), 182-198.

González et al., 2019; Assunção et al., 2020). Uma viscosidade elevada culmina a redução da eficiência da atomização, além de ocasionar a deposição de resíduos no motor (Lôbo, Ferreira, \& Cruz, 2009).

Tabile et al. (2009) descreve uma viscosidade de 14,5 cSt para o biodiesel das sementes de $R$. communis, sendo um valor fora dos limites permitidos pela portaria da Agência Nacional do Petróleo (2,5 a 5,5 cSt). A adequação da viscosidade do biodiesel pode ser realizada através da acetilação da hidroxila presente no ácido ricinoleico, sendo que já se estudou o uso de reações enzimáticas para este processo (Assunção et al., 2020). Todavia, ainda não há estudos sobre a acetilação do biodiesel de $R$. communis por via química.

O biodiesel produzido com esta oleaginosa tem grande potencial ao evitar a competição pela oleaginosas destinadas à alimentação humana (Osorio-González et al., 2019), sobretudo no Brasil, já que o governo prioriza a produção de oleaginosas que tragam maior impacto social, neste sentido a $R$. communis tem grande impacto na região nordeste do país devido a sua resistência a climas semiáridos (Ferreira \& Melo, 2019, Assunção, 2020).

A produção de biodiesel de $R$. communis é favorecida na região nordeste devido ao programa nacional de produção e uso de biodiesel instituído pela Lei n. ${ }^{\circ}$ 11.097/2005 (BRASIL, 2005), entretanto existe a dificuldade na adequação do biodiesel de $R$. communis às normas da ANP, principalmente por causa de sua viscosidade (César \& Batalha, 2011).

O biodiesel de $R$. communis possui propriedades interessantes, como a estabilidade oxidativa que pode auxiliar outros biodieseis com alta insaturação, neste sentido, é uma matéria prima importante para a diversificação da matriz energética dos países que possuem condições adequadas de cultivo para esta oleaginosa (Vieira, Nadaleti, \& Sarto, 2021).

A estabilidade oxidativa é vantajosa por aumentar o tempo de estocagem do biodiesel, entretanto é necessário considerar que o biodiesel sofre alterações aumentando sua viscosidade ao longo que se degrada, assim um biodiesel muito viscoso se distancia ainda mais das normas durante a estocagem (Suota et al., 2018), ou seja, a adequação da viscosidade é necessária para que o uso deste produto possa ocorrer em maior escala.

Diante do exposto, esta pesquisa visa analisar o efeito da acetilação por rota química para adequação do biodiesel do óleo das sementes de $R$. communis. Para isto, objetivou-se realizar a síntese do biodiesel, analisando características físico-químicas e realizando a acetilação com acompanhamento por meio de ressonância magnética nuclear (RMN).

\section{MATERIAL E MÉTODOS}

\subsection{Reagentes utilizados e obtenção do óleo de $R$. communis}

O óleo das sementes de $R$. communis utilizado neste estudo foi obtido industrialmente por um produtor regional da cidade de Teresina, estado de Piauí, Brasil. A matéria prima já havia passado pelas etapas de refino de degomagem, neutralização, desumidificação e filtração. A Tabela 1 descreve os reagentes químicos utilizados. 
Citação (APA): Silva, C. C. M. da, Moraes, L. C. K., Santos, J. R. dos, Jr, \& Castro, T. L. A. de (2021). Redução da viscosidade do biodiesel de Ricinus Communis L. (mamona) por acetilação química. Brazilian Journal of Production Engineering, 7(3), 182-198.

Tabela 1. Especificações dos reagentes utilizados

\begin{tabular}{ll}
\hline \multicolumn{1}{c}{ Reagentes } & \multicolumn{1}{c}{ Especificação } \\
\hline Álcool metílico & Absoluto, para análise, ACS (11871), peso molecular: 32,04, \\
& dosagem: min. 99,8\% \\
Hidróxido de potássio (pó) & Vetec P.A. - Peso molecular: 56,11, teor mínimo 85\% \\
Piridina & Vetec - Peso molecular: 79,10 \\
Anidrido acético & Vetec - Peso molecular: 102,09 \\
Sulfato de sódio anidro & Vetec - Peso molecular: 248,18 \\
\hline
\end{tabular}

As estruturas das moléculas e os mecanismos foram desenhadas através do software ChemDraw 18.1 (Perkin-Elmer, USA).

\subsection{Métodos de transesterificação dos lipídios}

O pré-tratamento do óleo é essencial para garantir que o biodiesel produzido se enquadre dentro das normas exigidas (Silva \& Fonseca, 2021). O óleo foi previamente desumidificado para retirar umidade residual absorvido na estocagem e transporte.

Foi realizado as análises de índices no óleo de acidez (NBR 14448) e saponificação (NBR 5834), alcalinidade livre, combinada e total (titulometria de neutralização), glicerina livre e combinada (AOCS Ca 14-56), viscosidade cinemática a $40^{\circ} \mathrm{C}$ (ASTMD 2270), teor de enxofre (NBR 15546), densidade a $20^{\circ} \mathrm{C}$ (NBR 14065), ponto de fulgor (NBR 14598), corrosividade ao cobre (NBR 14359), cinzas sulfatadas (NBR 9842), água e sedimentos (NBR 14647), resíduo de carbono (NBR 14318), ponto de entupimento a frio (NBR 14747), teor de $\mathrm{Ca}+\mathrm{Mg}$ e teor de $\mathrm{Na}+\mathrm{K}$ (NBR 15553). Todas análises foram realizadas em triplicata.

Após seu resfriamento espontâneo, este foi transesterificado por dois métodos distintos A e B. A proporção de óleo e álcool utilizada foi baseada no trabalho de Lima et al. (2007). O óleo $(500 \mathrm{~g})$ foi submetido à magnética sob aquecimento de $60^{\circ} \mathrm{C}$, paralelamente preparou-se uma solução de $100 \mathrm{~g}$ de metanol e $5 \mathrm{~g}$ de hidróxido de potássio, em seguida adicionou-se a solução ao óleo, deixou-se agitar por 30 minutos (amostra A) e por 60 minutos (amostra B).

Após o término da reação, realizando-se a separação das fases da mistura: O sobrenadante corresponde ao solvente rico em ésteres metílicos e uma fase sedimentada corresponde a glicerina dissolvida. Em sequência, realizou-se a destilação do sobrenadante, removendo o excesso de metanol. Com os valores exatos das massas de biodiesel, glicerina e metanol, cálculo se o rendimento da reação.

Para purificação dos ésteres metílicos de ácido graxo obtido, adicionou-se água aquecida a $40^{\circ} \mathrm{C}$ para remover resíduos do catalisador, glicerina livre e excesso de metanol, com aquecimento a $100^{\circ} \mathrm{C}$, obtendo um aspecto translúcido. Posteriormente o biodiesel foi avaliado físicoquimicamente.

\subsection{Acetilação dos ésteres metílicos}

Os dois tipos de ésteres metílicos (A e B) obtidos foram tratados com piridina ( $25 \mathrm{~g}$ ) e anidrido acético $(50 \mathrm{~g})$. A mistura foi mantida sob agitação durante 24 horas, decorrido este período a mesma foi vertida em $40 \mathrm{~mL}$ de água a temperatura de $10^{\circ} \mathrm{C}$, este procedimento foi repetido por sete vezes, no intuito de minimizar o excesso de piridina. $\mathrm{O}$ biodiesel foi desumidificado com sulfato de sódio anidro e em seguida com ar frio.

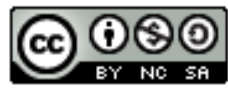


Citação (APA): Silva, C. C. M. da, Moraes, L. C. K., Santos, J. R. dos, Jr, \& Castro, T. L. A. de (2021). Redução da viscosidade do biodiesel de Ricinus Communis L. (mamona) por acetilação química. Brazilian Journal of Production Engineering, 7(3), 182-198.

Realizou-se medidas de viscosidade cinemática de biodiesel (A e B), antes e após a acetilação.

\subsection{Ressonância magnética nuclear}

$\mathrm{O}$ estudo de RMN de ${ }^{1} \mathrm{H} \mathrm{e}{ }^{13} \mathrm{C}$ concentrou-se somente no óleo vegetal e nos produtos (A e B) após a acetilação. Os espectros de $\mathrm{RMN}$ de ${ }^{1} \mathrm{He} \mathrm{e}^{13} \mathrm{C}$ em solução, foram obtidos em equipamento GEMINI - 300 BB, do Laboratório de tecnologia farmacêutica da Universidade Federal da Paraíba (UFPB). Os espectros foram obtidos utilizando $\mathrm{CDCl}_{3}$ (clorofórmio deuterado) como solvente.

\subsection{Viscosidade Cinemática}

As medidas de viscosidade foram feitas utilizando um viscosimétrico cinemático manual, utilizando tubo Cannon Fenske em banho térmico Koehler KV3000 de acordo com NBR 10441. A análise ocorreu em triplicata.

\section{RESULTADOS E DISCUSSÃO}

\section{1 Óleo da semente de $R$. communis}

O óleo das sementes de $R$. communis utilizado na síntese de biodiesel apresentou características físico-químicas similares e típicas as encontradas na literatura (Moreto \& Alves, 1986, Knothe \& Steidley, 2005; Elango et al., 2019). Na Tabela 2 são apresentados os valores de índice de acidez, índice de saponificação, viscosidade cinemática, teor de umidade e cor do óleo de $R$. communis utilizado.

Tabela 2. Parâmetros físico-químicos do óleo de $R$. communis

\begin{tabular}{cc}
\hline Parâmetros & Óleo de $\boldsymbol{R}$. communis \\
\hline Índice de acidez $(\mathrm{mg}$ de $\mathrm{KOH} / \mathrm{g}$ da amostra $\pm \mathrm{DP})$ & $0,9553 \pm 0,0192$ \\
Índice de saponificação $(\mathrm{mg}$ de $\mathrm{KOH} / \mathrm{g}$ da amostra $\pm \mathrm{DP})$ & $177,28 \pm 0,08$ \\
Viscosidade cinemática $\left(\mathrm{mm}^{2} / \mathrm{s} \pm \mathrm{DP}\right)$ & $245,05 \pm 0,01$ \\
Teor de umidade a $85^{\circ} \mathrm{C}(\%) \pm \mathrm{DP}$ & $0,35 \pm 0,07$ \\
Cor $(\mathrm{ASTM})$ & $0,5 \pm 0,1$ \\
\hline
\end{tabular}

$\mathrm{DP}=$ Desvio padrão.

O valor de índice de acidez é reflexo do processo de refino que neutralizou parte da quantidade dos ácidos graxos livres. Este valor é bem adequado à reação de transesterificação (Rovere, Rodrigues, \& Teleken, 2020). Segundo Azevedo e Lima (2001), o índice de saponificação do óleo de $R$. communis é da ordem de $180 \mathrm{mg} \mathrm{KOH/g}$. O índice de saponificação encontrado é condizente com o relatado na literatura.

Um parâmetro que apresenta valor bem característico do óleo de $R$. communis é a viscosidade cinemática, pois óleos como soja, babaçu e outros apresentam viscosidade em torno de 30 $\mathrm{mm}^{2} / \mathrm{s}$ (Lima et al., 2005). O valor obtido (Tabela 2) é elevado e isto influencia a viscosidade do biodiesel.

A Figura 1 apresenta o espectro de $\mathrm{RMN}$ de ${ }^{1} \mathrm{H}$ e a Figura 2 o espectro $\mathrm{RMN}$ de ${ }^{13} \mathrm{C}$ do óleo de R. communis. 
Citação (APA): Silva, C. C. M. da, Moraes, L. C. K., Santos, J. R. dos, Jr, \& Castro, T. L. A. de (2021). Redução da viscosidade do biodiesel de Ricinus Communis L. (mamona) por acetilação química. Brazilian Journal of Production Engineering, 7(3), $182-198$.

Figura 1. Espectro de $\mathrm{RMN}$ de ${ }^{1} \mathrm{H}$ do óleo da semente de $R$. communis refinado

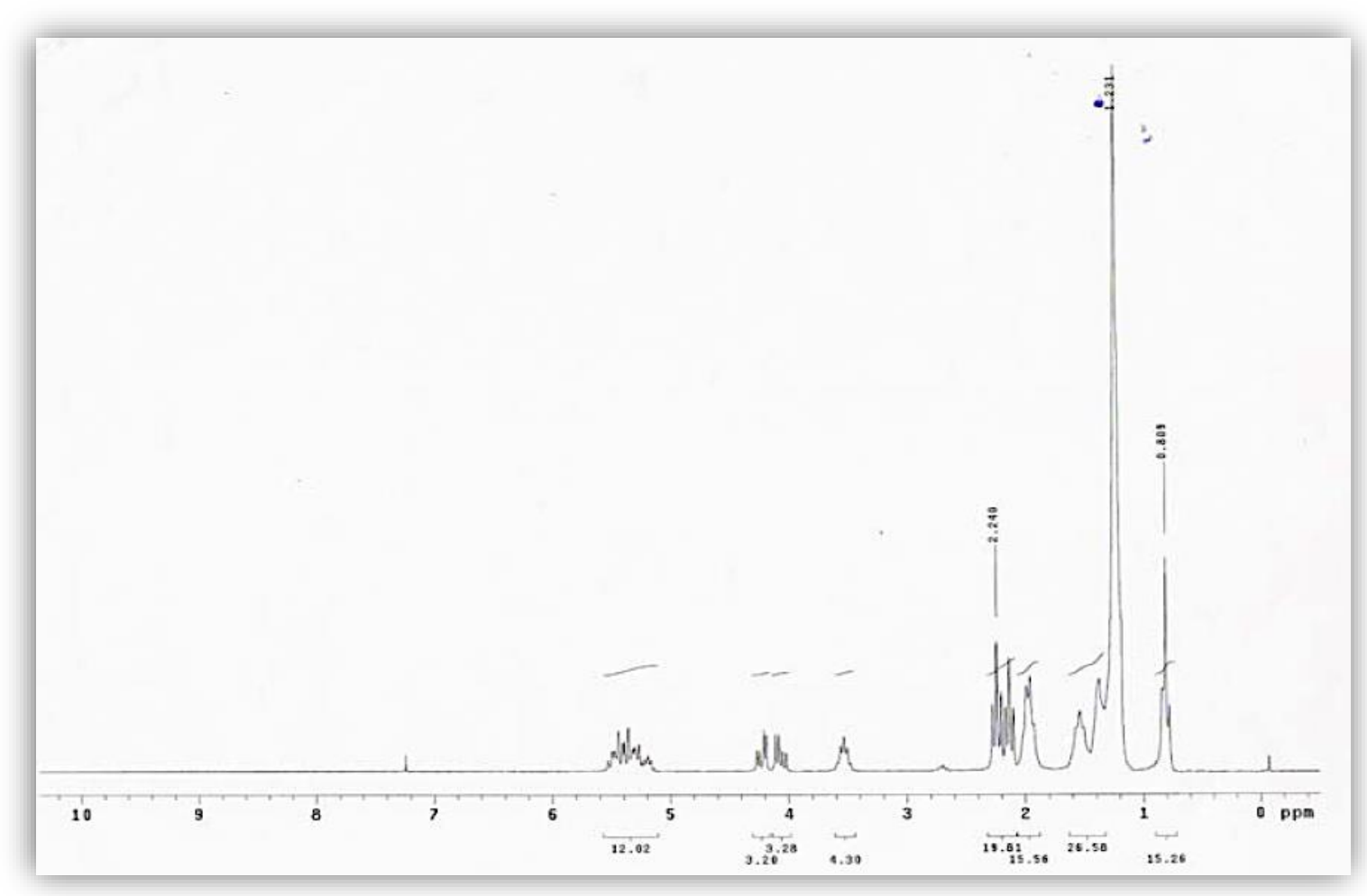

Fonte: Autores (2021)

Figura 2. Espectro $\mathrm{RMN}$ de ${ }^{13} \mathrm{C}$ do óleo da semente de $R$. communis refinado

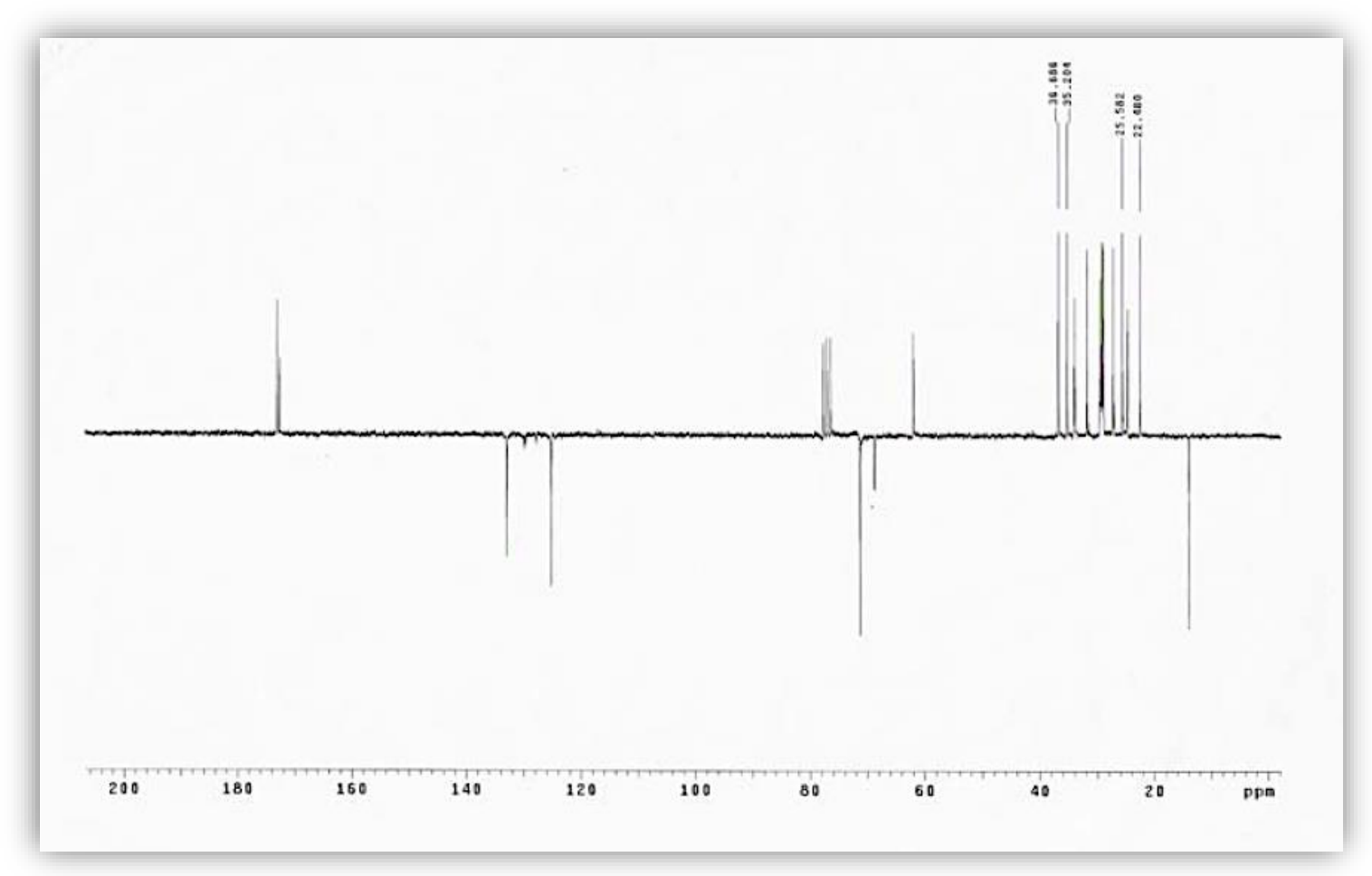

Fonte: Autores (2021)

Observa-se a predominância do ácido graxo majoritário, ácido ricnoleico. As atribuições características, seguem na Tabela 3.

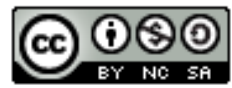


$-188-$

Citação (APA): Silva, C. C. M. da, Moraes, L. C. K., Santos, J. R. dos, Jr, \& Castro, T. L. A. de (2021). Redução da viscosidade do biodiesel de Ricinus Communis L. (mamona) por acetilação química. Brazilian Journal of Production Engineering, 7(3), 182-198.

Tabela 3. Atribuições de $\mathrm{RMN}{ }^{1} \mathrm{H}$ e ${ }^{13} \mathrm{C}$ do óleo de $R$. communis

\begin{tabular}{|c|c|c|c|}
\hline Posição & ${ }^{1} \mathrm{H}(\delta)$ & ${ }^{13} \mathrm{C}(\delta)$ & Multiplicidade \\
\hline$\alpha \alpha^{\prime}, \beta$ & $4,0-4,2$ & $62,1\left(\alpha \alpha^{\prime}\right), 69,0 \beta$ & $\mathrm{CH}_{2}$ (glicerol) \\
\hline 1 & - & 172 & $\mathrm{C}$ (carbonila) \\
\hline 2 & 1,96 & 35,94 & $\mathrm{CH}_{2}$ (ligado a carbonila) \\
\hline 3 & 1,57 & 22,48 & $\mathrm{CH}_{2}$ \\
\hline 4 & 1,54 & 24,62 & $\mathrm{CH}_{2}$ \\
\hline 5 & 1,51 & 25,58 & $\mathrm{CH}_{2}$ \\
\hline 6 & 1,40 & 27,05 & $\mathrm{CH}_{2}$ \\
\hline 7 & 1,23 & 27,23 & $\mathrm{CH}_{2}$ \\
\hline 8 & 2,2 & 24,03 & $\mathrm{CH}_{2}$ \\
\hline 9 & 5,2 & 125,22 & $\mathrm{CH}$ (instauração) \\
\hline 10 & 5,6 & 132,91 & CH (instauração) \\
\hline 11 & 2,26 & 35,94 & $\mathrm{CH}_{2}$ \\
\hline 12 & 3,48 & 71,34 & $\mathrm{CH}-\mathrm{OH}$ \\
\hline 13 & 1,2 & 35,20 & $\mathrm{CH}_{2}$ \\
\hline 14 & 1,23 & 28,89 & $\mathrm{CH}_{2}$ \\
\hline 15 & 1,23 & 28,99 & $\mathrm{CH}_{2}$ \\
\hline 16 & 1,23 & 29,23 & $\mathrm{CH}_{2}$ \\
\hline 17 & 1,4 & 29,44 & $\mathrm{CH}_{2}$ \\
\hline 18 & 0,8 & 13,9 & $\mathrm{CH}_{3}$ \\
\hline $\mathrm{OH}$ & 4,20 & - & - \\
\hline
\end{tabular}

Ao comparar com RMN ${ }^{1} \mathrm{H}$ de óleo de babaçu (Lima et al. 2005) com o obtido é possível observar a ausência dos sinais em 3,48 e 4,20 característicos da presença da hidroxila ligada ao C 12 do ácido ricinoleico do óleo de $R$. communis. A Figura 3 apresenta a estrutura do ácido ricinoleico obtida através da análise por RMN. Segundo Knoth (2001), é possível constatar que o biodiesel metílico de soja também não apresenta estes picos, enquanto que o espectro RMNH do biodiesel metílico de R. communis obtido por Torrentes-Espinoza et al. (2017) apresenta estes picos característicos da hidroxila.

Figura 3. Estrutura do ácido ricinoleico com as numerações dos carbonos

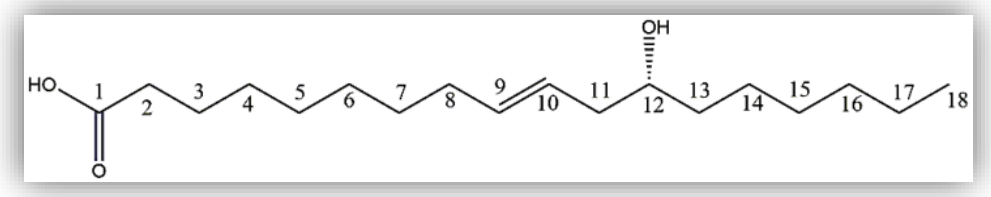

Fonte: Autores (2021)

\subsection{Rendimentos reacionais dos métodos de transesterificação.}

Os rendimentos reacionais de transesterificação dos métodos A $(91,0 \%)$ e B $(94,6 \%)$ foram satisfatórios e concordam com o obtido utilizando-se outros óleos. Lima et al. (2005), alcançou rendimentos da ordem de $90 \%$ para os óleos de soja, babaçu, tucum e macaúba adotando método similar ao deste trabalho, porém utilizando via etílica. O biodiesel metílico de Moringa oleífera apresenta rendimento de $99 \%$ (Oliveira et al., 2012), já Ventura, Alves e Santos(2010) obteve um rendimento de $72 \%$ para o biodiesel de $R$. communis por via metílica. O método B 
Citação (APA): Silva, C. C. M. da, Moraes, L. C. K., Santos, J. R. dos, Jr, \& Castro, T. L. A. de (2021). Redução da viscosidade do biodiesel de Ricinus Communis L. (mamona) por acetilação química. Brazilian Journal of Production Engineering, 7(3), 182-198.

apresentou um rendimento reacional superior ao A devido ao maior tempo aplicado na transesterificação, resultando assim, um teor de conversão maior de triglicerídeos em ésteres metílicos e glicerol.

\subsection{Características físico-químicas do biodiesel de $R$. communis}

As características dos biodieseis obtidos pelos diferentes métodos (A e B) são bastante concordantes entre si, tão quanto com a Resolução da Agência Nacional de Petróleo, Gás Natural e Biocombustíveis (ANP, 2014) (Tabela 4). O índice de acidez representa o teor de ácidos graxos livres presente no biodiesel, todos os resultados obtidos apresentaram-se abaixo do permitido pela ANP (2014) (0,50 mg de KOH/g da amostra) (Tabela 4).

Tabela 4. Parâmetros físico-químicos dos dois biodieseis A e B

\begin{tabular}{|c|c|c|c|c|}
\hline Parâmetros & ANP & Método & $\begin{array}{c}\text { Biodiesel } \\
\text { Amostra A } \\
\end{array}$ & $\begin{array}{c}\text { Biodiesel } \\
\text { Amostra B } \\
\end{array}$ \\
\hline Aspecto & LII & Visual & LII & LII \\
\hline $\begin{array}{c}\text { Índice de Acidez }\left(\mathrm{mg} \text { de } \mathrm{KOH} \mathrm{g} \mathrm{g}^{-1} \text { da }\right. \\
\text { amostra } \pm \mathrm{DP})\end{array}$ & 0,50 & ASTM D 664 & $0,226 \pm 0,045$ & $0,260 \pm 0,064$ \\
\hline Alcalinidade livre (meq $\left.\mathrm{g}^{-1} \pm \mathrm{DP}\right)$ & - & - & ND & ND \\
\hline Alcalinidade combinada (meq g $\left.\mathrm{g}^{-1} \pm \mathrm{DP}\right)$ & - & - & $0,049 \pm 0,001$ & $0,0452 \pm 0,001$ \\
\hline Teor de enxofre $(\mathrm{ppm} \pm \mathrm{DP})$ & 10 & ASTM D4294 & ND & ND \\
\hline Ponto de Fulgor $\left({ }^{\circ} \mathrm{C}\right)$ & 120,0 & ASTM D 93 & $>150$ & $>150$ \\
\hline Densidade a $20 \pm 0.01{ }^{\circ} \mathrm{C}\left(\mathrm{g} \mathrm{cm}^{3} \pm \mathrm{DP}\right)$ & 850 a 900 & ASTM D 4-052 & $0,92 \pm 0,01$ & $0,92 \pm 0,01$ \\
\hline Glicerina livre $(\%)$ & 0,02 & AOCS Ca14-56 & 0,06 & 0,02 \\
\hline Glicerina total (\%) & 0,25 & AOCS Ca14-56 & 0,89 & 0,42 \\
\hline Cor $(\mathrm{ASTM}) \pm \mathrm{DP}$ & - & - & $0,5 \pm 0,1$ & $0,5 \pm 0,1$ \\
\hline Água e sedimentos (\% volume) & 0,050 & ASTM D 2709 & $<0,05$ & $<0,05$ \\
\hline Corrrosividade ao cobre $3 \mathrm{~h}$ a $50^{\circ} \mathrm{C}$, Max & 1 & ASTM D 130 & 1 & 1 \\
\hline Teor de cinzas sulfatadas (\% massa) & 0,020 & ASTM D 874 & $<0,0010$ & $<0,0010$ \\
\hline Resíduo de carbono (\% massa) & 0,10 & ASTM D 189 & 0,003 & $<0,0010$ \\
\hline Ponto de entupimento a frio $\left({ }^{\circ} \mathrm{C}\right)$ & - & NBR 14747 & -1 & -2 \\
\hline $\mathrm{Na}+\mathrm{K}(\mathrm{ppm})$ & 10 & EN 14108 & 1,25 & 2,80 \\
\hline $\mathrm{Ca}+\mathrm{Mg}(\mathrm{ppm})$ & 10 & EN 14108 & 1,82 & 8,52 \\
\hline
\end{tabular}

ND = Não detectável.

O tempo reacional não induziu a modificação entre as amostras A e B para os parâmetros: Aspecto, Alcalinidade livre, teor de enxofre, ponto de fulgor, densidade, cor, água e sedimentos, corrosividade e teor de sulfatados (Tabela 4). O aumento do tempo de reação aumentou os parâmetros: Índice de acidez, ponto de entupimento a frio, quantidade de $\mathrm{Na}+\mathrm{K}$ e $\mathrm{Ca}+\mathrm{Mg}$ (Tabela 4).

O biodiesel apresenta teores de enxofre inferiores ao diesel (Fracetto, Fracetto, Feigl, Cerri, \& Neto, 2015). A ANP (2014) limitou o máximo de 10 ppm do teor enxofre para o biodiesel. O teor de enxofre e o fósforo do biodiesel são provenientes dos fosfolipídios presentes no óleo vegetal, todavia o processo de degomagem remove estes compostos (Lôbo, Ferreira \& Cruz, 2009). O óleo utilizado para a produção do biodiesel passou pelo pré-tratamento adequado, justificando a ausência de enxofre (Tabela 4).

A viscosidade cinemática e a densidade são inerrantes ao biodiesel de $R$. communis (Assunção et al., 2020). Obtiveram-se valores de viscosidade a partir de 14,0 cSt (Tabela 4) enquanto a ANP (2014) estabelece um valor entre 3,0 a 6,0 cSt, para que se tenha condições adequadas de 
Citação (APA): Silva, C. C. M. da, Moraes, L. C. K., Santos, J. R. dos, Jr, \& Castro, T. L. A. de (2021). Redução da viscosidade do biodiesel de Ricinus Communis L. (mamona) por acetilação química. Brazilian Journal of Production Engineering, 7(3), 182-198.

injeção do combustível nos motores que empregam diesel mineral. Mediante este quadro, revelou-se a necessidade da derivatização do biodiesel no intuito de inseri-lo nas especificações vigentes para viscosidade.

O ponto de fulgor é definido como sendo a menor temperatura na qual o biodiesel, ao ser aquecido sob condições controladas, gera uma quantidade de vapores que se inflamam (Silva \& Andrade, 2020). Tal parâmetro relacionado a inflamabilidade do produto, é um indicativo dos procedimentos de segurança a serem tomados durante o uso, transporte, armazenamento e manuseio do biodiesel (Lôbo, Ferreira, \& Cruz, 2009). Se o biocombustível for completamente isento de resíduos de álcool, seu ponto de fulgor será superior a temperatura ambiente, indicando que o produto não é inflamável nas condições normais de manuseio (Zuniga et al., 2011).

Os resultados encontrados para ponto de fulgor encontram-se acima do mínimo permitido pela ANP (2014) $\left(120^{\circ} \mathrm{C}\right)$, assim como acima dos pontos de fulgor encontrados por Lima et al. (2005) para outros biodieseis. Tais resultados são explicados devido à composição atípica do biodiesel de $R$. communis (elevada constituição de ricinoleato de metila) que possui uma cadeia longa (18 Carbonos) e uma hidroxila no $12^{\circ}$ Carbono. Isto garante o aumento de temperatura e demonstra a direta influência da composição química do óleo em relação aos parâmetros físicoquímicos apresentados pelo biodiesel. Uma vantagem desta característica é que quanto maior o ponto de fulgor mais se torna seguro o transporte e armazenamento do biodiesel.

Os valores de glicerina livre para o biodiesel obtido pelo método apresentaram-se fora da especificação segundo a portaria 45 da ANP (2014). Estes resultados são reflexos da forte interação dos ésteres com o glicerol, mas o que é perfeitamente corrigível na etapa de purificação com a utilização de adsorventes (Lima et al., 2005). Todos os valores encontrados para glicerina total, encontram-se acima do limite permitido pela ANP (2014), tais resultados mostram que as reações em escala laboratoratorial não são tão eficientes devido as agitações utilizadas no intuito de aumentar a superfície de contato entre os reagentes. O aumento do tempo reacional reduziu os teores de glicerina livre e total (Tabela 4).

Os resultados do teor de água e sedimentos dos dois tipos de biodiesel estudados, encontramse abaixo do limite estabelecido pela ANP (2014), mostrando assim uma eficiente desumidificação. Tal determinação em amostras de biodiesel visa controlar a presença de contaminantes sólidos e água, já que os sólidos podem reduzir a vida útil dos filtros dos veículos e a presença de água pode gerar contaminações microbianas e promover hidrólise do biodiesel (Lôbo, Ferreira, \& Cruz, 2009; Parente, 2003; ANP, 2014).

A corrosividade ao cobre, trata-se da avaliação do caráter corrosivo do produto, indicando o potencial de corrosividade do diesel, no que diz respeito às peças metálicas e confeccionadas em ligas de cobre, que se encontram presentes nos sistemas de combustível dos veículos e equipamentos, além das instalações de armazenamento, estando associado à presença de enxofre (Lôbo, Ferreira, \& Cruz, 2009). Pelas normas da ANP, a corrosividade ao cobre máxima permitida são os níveis 1, 1a e 1b na lâmina padrão da ASTM, segundo os padrões do método-teste. Como observa-se na Tabela 5, os biodieseis apresentaram padrão 1 (Parente, 2003; ANP, 2014). 
Citação (APA): Silva, C. C. M. da, Moraes, L. C. K., Santos, J. R. dos, Jr, \& Castro, T. L. A. de (2021). Redução da viscosidade do biodiesel de Ricinus Communis L. (mamona) por acetilação química. Brazilian Journal of Production Engineering, 7(3), 182-198.

Tabela 5. Atribuições de $\mathrm{RMN}{ }^{1} \mathrm{H}$ e ${ }^{13} \mathrm{C}$ do biodiesel de mamona acetilado A

\begin{tabular}{|c|c|c|c|}
\hline Posição & ${ }^{1} \mathrm{H}(\boldsymbol{\delta})$ & ${ }^{13} \mathrm{C}(\delta)$ & Multiplicidade \\
\hline 1 & - & 174 & C (carbonila) \\
\hline 2 & 1,96 & 35,94 & $\mathrm{CH}_{2}$ (ligado a carbonila) \\
\hline 3 & 1,57 & 22,48 & $\mathrm{CH}_{2}$ \\
\hline 4 & 1,54 & 24,62 & $\mathrm{CH}_{2}$ \\
\hline 5 & 1,51 & 25,58 & $\mathrm{CH}_{2}$ \\
\hline 6 & 1,40 & 27,05 & $\mathrm{CH}_{2}$ \\
\hline 7 & 1,23 & 27,23 & $\mathrm{CH}_{2}$ \\
\hline 8 & 2,2 & 24,03 & $\mathrm{CH}_{2}$ \\
\hline 9 & 5,20 & 124,62 & CH (instauração) \\
\hline 10 & 5,60 & 132,54 & CH (instauração) \\
\hline 11 & 2,15 & 35,15 & $\mathrm{CH}_{2}$ \\
\hline 12 & 4,77 & 71,34 & $\mathrm{CH}-\mathrm{OH}$ \\
\hline 13 & 1,2 & 35,20 & $\mathrm{CH}_{2}$ \\
\hline 14 & 1,20 & 28,89 & $\mathrm{CH}_{2}$ \\
\hline 15 & 1,20 & 28,99 & $\mathrm{CH}_{2}$ \\
\hline 16 & 1,20 & 29,23 & $\mathrm{CH}_{2}$ \\
\hline 17 & 1,4 & 36,67 & $\mathrm{CH}_{2}$ \\
\hline 18 & 0,8 & 13,9 & $\mathrm{CH}_{3}$ \\
\hline $\mathrm{H}_{3} \mathrm{CCO}$ & 1,92 & 20,98 & $\mathrm{CH}_{3}$ (acetato) \\
\hline $\mathrm{H}_{3} \mathrm{CO}$ & 3,55 & 51,18 & $\mathrm{CH}_{3}$ (ester) \\
\hline
\end{tabular}

Resíduo de Carbono corresponde a quantidade de glicerídeos, água livre, sabões, sobras de catalisador e insaponificáveis determinam a quantidade de resíduo carbonáceo deixado pela amostra em análise (Lôbo, Ferreira, \& Cruz, 2009). Destaca-se os valores obtidos para os biodieseis, encontrou-se o maior valor para o biodiesel método A (0,003\%). (Parente, 2003; ANP, 2014).

A presença de metais pode ocasionar corrosão e problemas mecânicos, sendo que o sódio e o potássio podem ser incorporados no biocombustível pela utilização de hidróxidos de sódio e potássio como catalizadores (Barros, Oliveira, Magalhães, \& Villa, 2012). Quanto aos valores de alcalinidade, não há normatização pela portaria 45 da ANP (2014), entretanto eles refletem diretamente no teor de sódio (Na) e potássio (K) no biodiesel (Parente, 2003; ANP, 2014).

Os resultados de ponto de entupimento a frio como pode ser observado na Tabela 5, foram abaixo de $0{ }^{\circ} \mathrm{C}$, o que demonstra a viabilidade do uso de biodiesel de mamona em lugares de clima frio, não necessitando da adição de anticongelantes. $O$ ponto de entupimento a frio é um parâmetro físico-químico que representa a temperatura em que o fluido deixa de escoar, mantem-se uma correlação entre este parâmetro e a constituição química da matéria-prima, pois um elevado teor de ácidos graxos saturados resultara num elevado ponto de entupimento, devido ao elevado ponto de fusão dos mesmos (Singh, Sharma, Soni, Sharma, \& Kumari, 2019, Sia et al., 2020). Pela legislação vigente, a mistura óleo diesel / biodiesel utilizada deverá obedecer aos limites estabelecidos para ponto de entupimento de filtro a frio constantes da 
Citação (APA): Silva, C. C. M. da, Moraes, L. C. K., Santos, J. R. dos, Jr, \& Castro, T. L. A. de (2021). Redução da viscosidade do biodiesel de Ricinus Communis L. (mamona) por acetilação química. Brazilian Journal of Production Engineering, 7(3), 182-198.

especificação vigente da ANP (2014) de óleo diesel automotivo (Parente, 2003; ANP, 2014; Menezes \& Bernado, 2017).

O biodiesel de $R$. communis apresentou propriedades físico químicas adequadas, com exceção da viscosidade e densidade, assim como o previsto.

\subsection{Acetilação dos ésteres metílicos}

A reação de acetilação teve como intuito substituir o hidrogênio da hidroxila dos ésteres ricinoleicos por um grupamento acetato é uma opção de modificação química que pode levar uma a redução das interações entre as cadeias destes ésteres.

Os espectros de $\mathrm{RMN}$ de ${ }^{1} \mathrm{H}$ e ${ }^{13} \mathrm{C}$ mostram que houve uma redução quantitativa dos grupamentos hidroxilas presentes no biodiesel. Na Figura 4 encontra-se a estrutura do éster metílico de ácido ricinoleico acetilado. Através da Figura 5a e 5c (Espectros de $\mathrm{RMN}$ de $\left.{ }^{1} \mathrm{H}\right)$, observa-se que o biodiesel obtido pelo método A, a reação de transesterificação foi apenas parcial, fato confirmado pelos sinais entre 4,0 e 4,2, característicos de sinais de hidrogênio carboxílicos $\left(\mathrm{CHO}\right.$ e $\left.\mathrm{CH}_{2} \mathrm{O}\right)$.

Figura 4. Estrutura do ácido ricinoleico após a reação de acetilação

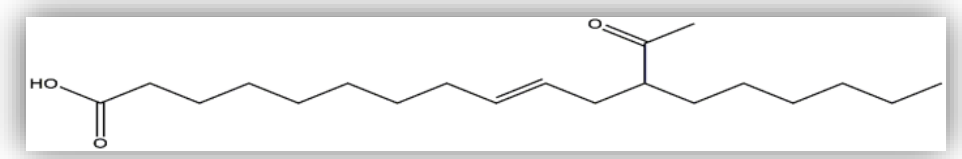

Fonte: Autores (2021)

Figura 5. RMN ${ }^{1} \mathrm{H}$ e ${ }^{13} \mathrm{C}$ DEPT do biodiesel de castor oil após acetilação: (a) RMN ${ }^{1} \mathrm{H}$ do método $\mathrm{A}$; (b) ${ }^{13} \mathrm{C}$ DEPT do método $\mathrm{A}$; (c) $\mathrm{RMN}{ }^{1} \mathrm{H}$ do método $\mathrm{B}$; and (d) ${ }^{13} \mathrm{C}$ DEPT do método B.

a)

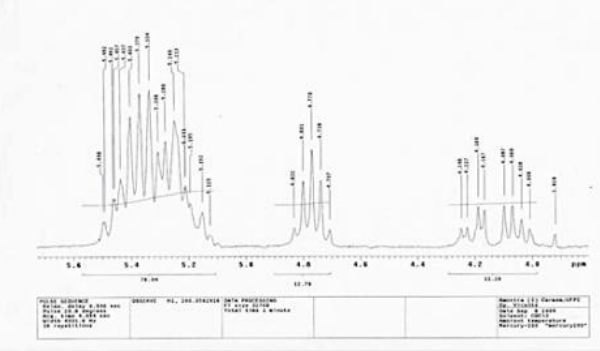

c)

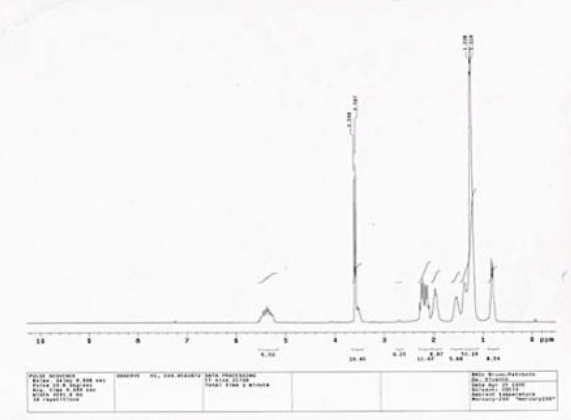

b)

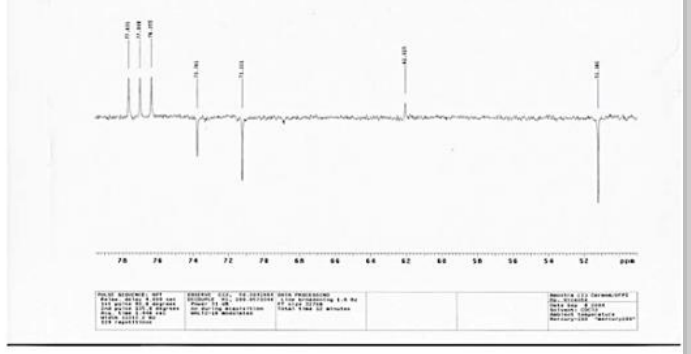

d)

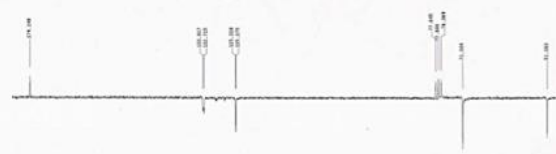

Fonte: Autor (2021) 

de Ricinus Communis L. (mamona) por acetilação química. Brazilian Journal of Production Engineering, 7(3), 182-198.

Foi observado o sinal do $\mathrm{H}-12$ do produto acetilado tal qual no biodiesel de partida, contudo através do espectro de ${ }^{13} \mathrm{C}$ (Figura $5 \mathrm{~b}$ e $5 \mathrm{~d}$ ) observa-se sinais típicos de acetilação, como a carbonila do acetato $(169 \delta)$ e o $\mathrm{CH}_{3}(20 \delta)$, introduzidos através da reação de acetilação. As atribuições características, seguem na Tabela 5.

A Figura 5 demonstra os espectros de $\mathrm{RMN}{ }^{1} \mathrm{H} \mathrm{e}{ }^{13} \mathrm{C}$ dos biodieseis obtidos pelos métodos A e $\mathrm{B}$, observou-se que a reação de transesterificação foi mais eficiente pelo método $\mathrm{B}$ em relação ao $\mathrm{A}$, pois a intensidade dos sinais na região entre 4,0 e 4,2 característicos de hidrogênio da parte glicérica da molécula do óleo vegetal, foi bem menor. Os espectros de ${ }^{1} \mathrm{H} \mathrm{e}{ }^{13} \mathrm{C}$, observase sinais típicos de acetilação, como a carbonila do acetato $(169 \delta)$ e o $\mathrm{CH}_{3}(20 \delta)$, introduzidos através da reação de acetilação.

Na Tabela 6, encontram-se os valores de viscosidade cinemática dos biodieseis acetilados das amostras A e B, é notoriamente visível a redução percentual que a reação de acetilação ocasionou na redução da viscosidade. Estas derivatizações ocasionaram modificações molecular na cadeia do ricinoleato de metila (Figura 4), responsável pela elevada viscosidade, resultando em produtos menos viscosos devido a redução das interações moleculares anteriores.

Tabela 6. Comparativo das viscosidades dos dois tipos de biodiesel obtidos

\begin{tabular}{|c|c|c|c|}
\hline \multirow{2}{*}{ Método de acetilação } & \multicolumn{2}{|c|}{ Viscosidade cinemática $\left(\mathrm{mm}^{2} / \mathrm{s} \pm \mathrm{DP}\right)$} & \multirow{2}{*}{$\begin{array}{c}\text { Redução da viscosidade } \\
(\%)\end{array}$} \\
\hline & Antes da acetilação & Após a acetilação & \\
\hline 30 minutos $(\mathrm{A})$ & $17,90 \pm 0,01$ & $12,45 \pm 0,01$ & 30,45 \\
\hline 60 minutos $(\mathrm{B})$ & $14,88 \pm 0,01$ & $10,02 \pm 0,01$ & 32,66 \\
\hline
\end{tabular}

A amostra A obteve uma viscosidade superior a amostra B (Tabela 6), apesar de apresenta um rendimento inferior. A redução da viscosidade após a acetilação foi superior para amostra B, mas mesmo assim, a amostra B apresentou a menor viscosidade após a acetilação. Uma hipótese é de que o tempo reacional tenha interferido na proporção de ácido ricinoleico obtido em relação aos demais ésteres de ácido graxo. Porém, os resultados não são conclusivos quanto ao motivo do método com maior tempo reacional produzir um biodiesel menos viscoso e mais suscetível a acetilação.

Os espectros de RMN de ${ }^{1} \mathrm{H}$ e ${ }^{13} \mathrm{C}$, confirmam a ocorrência destas reações (Figura 5a, 5b, 5c e 5d). A Figura 6 apresenta a reação global do processo de acetilação do ácido ricinoleico.

Figura 6. Reação global da acetilação do ácido ricinoleico

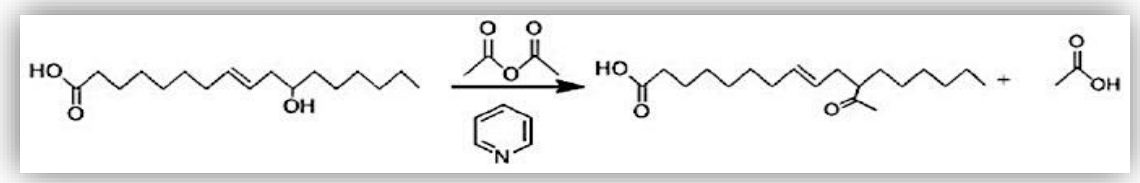

Uma proposta mecanística de reação para a acetilação do ácido ricinoleico está demonstrada na Figura 7. Neste mecanismo a piridina atua como catalisador, com a adição de água fria removendo o ácido carboxílico formado após a reação. 
Figura 7. Proposta de mecanismo para a reação de acetilação do ácido ricinoleico

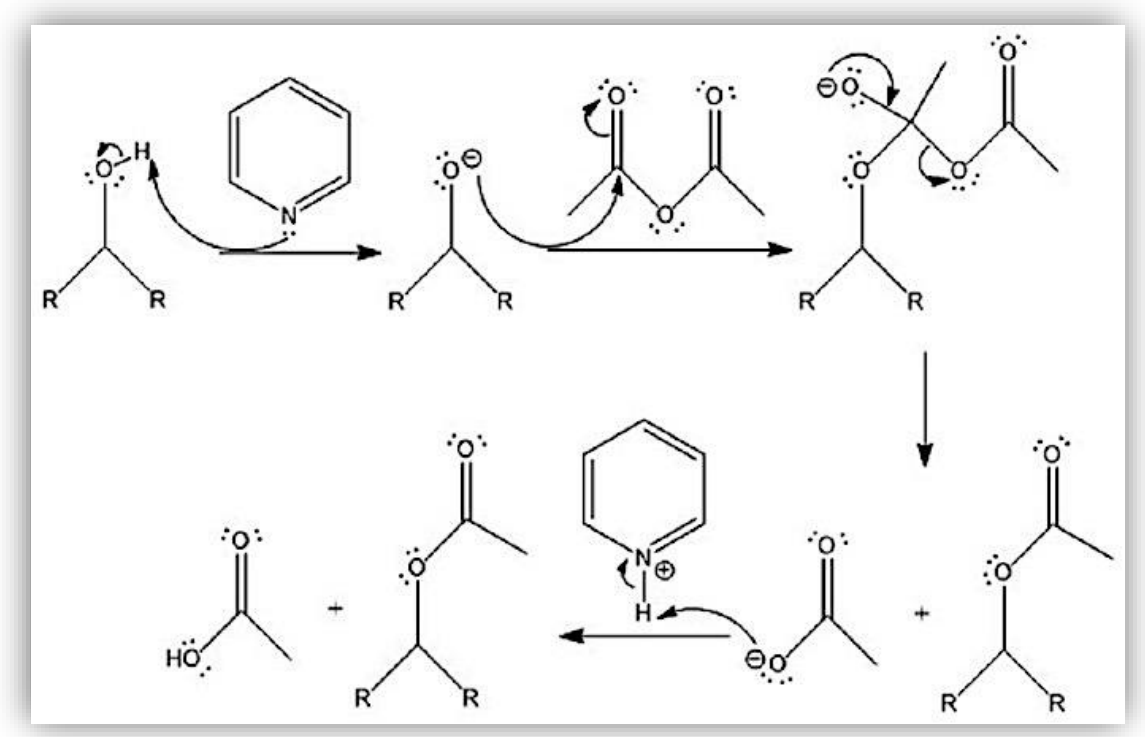

A viscosidade está associada a diversas propriedades importantes do combustível, como a atomização, pulverização e combustão, com altas viscosidade podendo ocasionar entupimento e retardamento da combustão (Erdoğana, Balki, \& Sayin, 2020). A viscosidade obtida ainda está fora das especificações da ANP (2014), contudo a redução da viscosidade permite adições de concentrações superiores de biodiesel em diesel, pois segundo Ventura et al. (2018), o biodiesel é miscível em diesel e o uso de blendas de biodiesel em diesel é comum. Neste sentido, um biodiesel de viscosidade superior é mais limitado para a utilização em blendas com diesel (Tat \& Van Gerpen, 1999).

Assunção et al. (2020) utilizou a rota enzimática, reduzindo a viscosidade do biodiesel de $R$. communis de 15,4 para 9,8 $\mathrm{mm}^{2} / \mathrm{s}$ utilizando a enzima Amano Lipase OS imobilizada. Todavia a viscosidade do método B apresentou uma viscosidade final semelhante aos obtidos por Assunção et al. (2020), indicando o método B de produção associado com a acetilação química é viável.

\section{CONCLUSÃO}

Dentro do analisado, o biodiesel de $R$. communis acetilado por rota química apresentou uma redução significativa de viscosidade, ampliando as possibilidades de blendas com diesel e se apresentando como uma alternativa para ampliar o uso desta matéria prima que tem impacto socioeconômico em regiões semiáridas que não compete com a produção alimentícia.

\section{REFERENCIAS}

Agência Nacional do Petróleo, Gás Natural e Biocombustíveis. (2014). Resolução ANP $N^{o} 45$ de 25/08/2014. Dispõe sobre a especificação do biodiesel contida no Regulamento Técnico ANP $n^{\circ} 3$ de 2014 e as obrigações quanto ao controle da qualidade a serem atendidas pelos diversos agentes econômicos que comercializam o produto em todo o território nacional. Diário da união: Brasília. 
Citação (APA): Silva, C. C. M. da, Moraes, L. C. K., Santos, J. R. dos, Jr, \& Castro, T. L. A. de (2021). Redução da viscosidade do biodiesel de Ricinus Communis L. (mamona) por acetilação química. Brazilian Journal of Production Engineering, 7(3), 182-198.

Assunção, J. C. C.; Valdevino, F. I. S. P., \& Rodrigues, F. E. A. (2020). Acetilação enzimática do biodiesel de Ricinus communis L. (mamona) visando a redução de sua viscosidade. Conexões Ciência e Tecnologia 14(3), 84-93. https://doi.org/10.21439/conexoes.v14i3.1459

Azevedo, D. M. P., \& Lima, E. F. (2001). Agronegócio da mamona no Brasil. Brasília: Embrapa Informação Tecnológica.

Borges, I. B., Faria, W. O., Pereira, E. C., Pureza, M. S., Siquara, L. L., Freitas, R. R., \& Porto, P. S. S. Programa jovens e meninas fazendo engenharia:cultivo de biomassa microalgal. Brazilian Journal of Production Engineering 2(2), 14-19. https://doi.org/10.0001/v2n3_nt01

Barros, A. I., Oliveira, A. P., Magalhães, M. R. L., \& Villa, R. D. (2012). Determination of sodium and potassium in biodiesel by flame atomic emission spectrometry, with dissolution in ethanol as a single sample preparation step. Fuel 93, 381-384. https://doi.org/10.1016/j.fuel.2011.08.060

Barros, L. H. C.; Purificação, M. C., Campanha, N., Silva, T. F. H., \& Santos, A. G. (2020). Biodiesel do óleo da semente de pinha produzido por reação via aquecimento e ultrassom. South American Journal of Basic Education, Technical and Technological 7(1), 94-107. Recuperado de https://periodicos.ufac.br/index.php/SAJEBTT/article/view/3316

Brasil. Lei $n^{o}$ 11.097, de 13 de janeiro de 2005. Dispõe sobre a introdução do biodiesel na matriz energética brasileira. Diário Oficial da República Federativa do Brasil, Brasília, DF, 14 jan. 2005.

Cangemi, J. M., Santos, A. M., \& Neto, S. C. (2010). A revolução verde da mamona. Química Nova na Escola 32(1), 1-6. Recuperado de http://qnesc.sbq.org.br/online/qnesc32_1/02-QS1209.pdf

Chechetto, R. G., Siqueira, R., \& Gamero, C. A. (2010). Balanço energético para a produção de biodiesel pela cultura da mamona (Ricinus communis L.). Revista Ciência Agronômica 41(4), 546-553. https://doi.org/10.1590/S1806-66902010000400006

César, A. S., \& Batalha, M. O. (2011). Análise dos direcionadores de competitividade sobre a cadeia produtiva de biodiesel: o caso da mamona. Produção 21(3), 484-497. https://doi.org/10.1590/S0103-65132011005000039

Costa, L. G., Sitoe, B. V., Santos, D. Q., \& Neto, W. B. (2020). Quantificação do teor de biodiesel de crambe em misturas com diesel utilizando espectroscopia MIR e seleção de variáveis. Química Nova 43(6), 723-728. https://doi.org/10.21577/0100-4042.20170554

Das, M., Sarkar, M., Datta, A., \& Santra, A. K. (2018). An experimental study on the combustion, performance and emission characteristics of a diesel engine fuelled with dieselcastor oil biodiesel blends. Renewable Energy 119, 174-184. https://doi.org/10.1016/j.renene.2017.12.014

Elango, R. K., Sathiasivan, K., Muthukumaran, C., Thangavelu, V., Rajesh, M., \& Tamilarasan, K. (2019). Transesterification of castor oil for biodiesel production: Process optimization and characterization. Microchemical Journal 145(1), 1162-1168. https://doi.org/10.1016/j.microc.2018.12.039

Erdoğana, S., Balki, M. K., \& Sayin, C. (2020). The effect on the knock intensity of high viscosity biodiesel use in a DI diesel engine. Fuel 253, 1162-1167. https://doi.org/10.1016/j.fuel.2019.05.114 
Citação (APA): Silva, C. C. M. da, Moraes, L. C. K., Santos, J. R. dos, Jr, \& Castro, T. L. A. de (2021). Redução da viscosidade do biodiesel de Ricinus Communis L. (mamona) por acetilação química. Brazilian Journal of Production Engineering, 7(3), 182-198.

Ferreira, R. S., \& Melo, A. S. (2019). Análise das fontes de crescimento do valor bruto da produção da mamona no período de 1990 a 2016. Revista em Agronegócio e Meio Ambiente 12(2), 487-513. https://doi.org/10.17765/2176-9168.2019v12n2p487-513

Fracetto, F. J. C., Fracetto, G. G. M., Feigl, B. J., Cerri, C. C., \& Neto, M. S. (2015). Emissões de gases de efeito estufa na produção de mamona e de seus subprodutos. Revista Caatinga 28(4), 90-98. https://doi.org/10.1590/1983-21252015v28n410rc

Keera, S. T., El Sabagh, S. M., \& Taman, A. R. (2018). Castor oil biodiesel production and

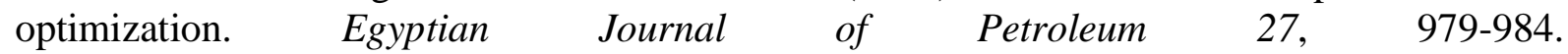
https://doi.org/10.1016/j.ejpe.2018.02.007

Knothe, G. (2001). Determining the blend level of mixtures of biodiesel with conventional diesel fuel by fiber-optic near-infrared spectroscopy and ${ }^{1} \mathrm{~h}$ nuclear magnetic resonance spectroscopy. Journal of the American Oil Chemists' Society, 78(10), 1025-1028. https://doi.org/10.1007/s11746-001-0382-0

Knothe, G., \& Steidley, K. R. (2005). Kinematic viscosity of biodiesel fuel components and related compounds. Influence of compound structure and comparison to petrodiesel fuel components. Fuel 84, 1059-1065. https://doi.org/10.1016/j.fuel.2005.01.016

Konradt-Moraes, L. C., Oliveira Junior, J. P., Cardoso, C. A. L., \& Vieira, S. C. H. (2019). Extração e quantificação do teor de lipídios de Microalgas de Piscicultura visando a produção de Biodiesel. In: Santos, F. (Org.), Meio Ambiente em Foco, (Vol. 9, Chap. 11, pp. 99-105). Belo Horizonte: Poisson.

Lima, R. L. S., Severino, L. S., Sampaio, L. R., Sofiatti, V., Gomes, J. Á., \& Beltrão, N. E. M. (2011). Blends of castor meal and castor husks for optimized use organic fertilizer. Industrial Crops and Products 33(2), 364-368. https://doi.org/10.1016/j.indcrop.2010.11.008

Lima, J. R. O., Silva, R. B., Silva, C. C. M., Santos, L. S. S., Santos Júnior, J. R., Moura, E. M., \& Moura, C. V. R. (2005). Biodiesel de babaçu (Orbignya sp.) obtido por via etanólica. Química Nova 30(3), 600-603. https://doi.org/10.1590/S0100-40422007000300019

Lôbo, I. P., Ferreira, S. L. C., \& Cruz, R. S. (2009). Biodiesel: Parâmetros de qualidade e métodos analíticos. Química Nova 32(6), 1596-1608. https://doi.org/10.1590/S0100$\underline{40422009000600044}$

Menezes, F. B. J., \& Bernardo, A. N. (2017). Biodisel No Ceará: Uma perspectiva desenvolvimentista e divulgatória. Revista Gestão \& Sustentabilidade Ambiental 6(1), 233-250. http://dx.doi.org/10.19177/rgsa.v6e12017233-250

Moreto, E., \& Alves, R. F. (1986). Óleos e gorduras vegetais: processamento e análises. Florianópolis: Editora da UFSC.

Oliveira, D. S., Fonseca, X. D. S., Farias, P. N., Bezerra, V. S., Pinto, C. H. C., Souza, L. D., Santos, A. G. D., \& Matias, L. G. O. (2012). Obtenção do biodiesel através da transesterificação do óleo de Moringa Oleífera Lam. Holos 28(1), 49-61. https://doi.org/10.15628/holos.2012.803

Parente, J. S. E. (2003). Biodiesel, o livro: Aventura tecnológica num país engraçado. Fortaleza: Tecbio.

Oliveira, C. Y. B., D'alessandro, E. B., Antoniosi Filho, N. R., Lopes, R. G., \& Derner, R. B. (2021). Synergistic effect of growth conditions and organic carbon sources for improving biomass production and biodiesel quality by the microalga Choricystis minor var. minor. $\begin{array}{lllll}\text { Science } & \text { of } & \text { Thetal } 143476 .\end{array}$ https://doi.org/10.1016/j.scitotenv.2020.143476 
Citação (APA): Silva, C. C. M. da, Moraes, L. C. K., Santos, J. R. dos, Jr, \& Castro, T. L. A. de (2021). Redução da viscosidade do biodiesel de Ricinus Communis L. (mamona) por acetilação química. Brazilian Journal of Production Engineering, 7(3), 182-198.

Oliveira Junior, J., Konradt-Moraes, L. C., Castro, T. L. A., \& Santos, M. S. M. (2021). Produção de biodiesel com Salvinia auriculata cultivada com biossólidos ou vinhaça. In: Maziero, R. (Org.), Engenharias: Tendências e Inovações (Chap. 10, pp.117-128). Belo Horizonte: Synapse Editora.

Osorio-González, C. S., Gómez-Falcon, N., Sandoval-Sales, F., Saini, R., Brar, S. K., \& Ramírez, A. A. (2019). Production of biodiesel from castor oil: A review. Energies 13(10), e2467. https://doi.org/10.3390/en13102467

Ozcanli, M., Akar, M. A., Calik, A., \& Serin, H. (2017). Using HHO (Hydroxy) and hydrogen enriched castor oil biodiesel in compression ignition engine. International Journal of Hydrogen Energy 42(36), 23366-23372. https://doi.org/10.1016/j.ijhydene.2017.01.091

Postaue, N., Konradt-Moraes, L. C., \& Asmus, R. M. F. (2019). Chorume como fonte de nutriente na produção da biomassa microalgal. Revista E-Xacta 12(2), 11-19. https://doi.org/10.18674/exacta.v12i2.2746

Rana, M., Dhamija, H., Prashar, B., \& Sharma, S. (2012). Ricinus communis L. - A Review. International Journal of PharmTech Research 4(4), 1706-1711. Recuperado de https://sphinxsai.com/2012/oct-dec/Pharmpdf/PT=48(1706-1711)OD12.pdf

Rizzardo, R. A. G., Milfont, M. O., Silva, S. E. M., \& Freitas, B. M. (2012). Apis mellifera pollination improves agronomic productivity of anemophilous castor bean (Ricinus communis). Anais da Academia Brasileira de Ciências 84(4), 1137-1145. https://doi.org/10.1590/S0001$\underline{37652012005000057}$

Rovere, B. O., Rodrigues, J. H., \& Teleken, J. G. (2020). Redução do índice de acidez através da neutralização e esterificação para produção de biodiesel. Brazilian Journal of Development 6(5), 24678-24686. https://doi.org/10.34117/bjdv6n5-064

Souta, M. J., Simionatto, E. L., Scharf, D. R., Motta, V., Moser, D., Oliveira, L. B., Pedroso, L. R. M., Wisniewski Junior, A., Wiggers, V. R., Botton, V., \& Meier, H. F. (2018). Avaliação de características de biodieseis de fontes alternativas submetidos a condições de armazenagem diferenciada. Química nova 41(6), 648-655. http://dx.doi.org/10.21577/0100-4042.20170222

Sia, C. B., Kansedo, J., Tan, Y. H., \& Lee, K. T. (2020). Evaluation on biodiesel cold flow properties, oxidative stability and enhancement strategies: A review. Biocatalysis and Agricultural Biotechnology 24, e101514. https://doi.org/10.1016/j.bcab.2020.101514

Singh, D., Sharma, D., Soni, S. L., Sharma, S., \& Kumari, D. (2019). Chemical compositions, properties, and standards for different generation biodiesels: A review. Fuel 253, 60-71. https://doi.org/10.1016/j.fuel.2019.04.174

Silva, C. C. M., \& Fonseca, G. G. (2021). Avaliação de técnicas de refino de óleo reciclado para a produção biodiesel. Brazilian Journal of Production Engineering 7(2), 169-176. https://doi.org/10.47456/bjpe.v7i2.35513

Silva, C. C. M., Carvalho, N. L. C., \& Fonseca, G. G. (2021). Indústrias produtoras de biodiesel: destinação correta aos efluentes através de implantação de políticas de produção mais limpa (P+L). Recima21 - Revista científica multidisciplinar 2(4), e24265. https://doi.org/10.47820/recima21.v2i4.265

Silva, G. C. R., \& Andrade, M. H. C. (2020). Criação de banco de dados para simulação da produção de biodiesel. parte 2: Estimativa de propriedades termofísicas do biodiesel. The Journal of Engineering and Exact Sciences 6(2), 179-188. https://doi.org/10.18540/jcecvl6iss2pp0179-0188 
Citação (APA): Silva, C. C. M. da, Moraes, L. C. K., Santos, J. R. dos, Jr, \& Castro, T. L. A. de (2021). Redução da viscosidade do biodiesel de Ricinus Communis L. (mamona) por acetilação química. Brazilian Journal of Production Engineering, 7(3), 182-198.

Singh, D., Sharma, D., Soni, S. L., Sharma, S., Sharma, P. K., \& Jhalani, A. (2020). A review on feedstocks, production processes, and yield for different generations of biodiesel. Fuel 262, e116553. https://doi.org/10.1016/j.fuel.2019.116553

Tabile, R. A., Lopes, A., Dabdoub, M. J., Camara, F. T., Furlani, C. E. A., \& Silva, R. P. (2009). Biodiesel de mamona no diesel interior e metropolitano em trator agrícola. Engenharia Agrícola 29(3), 412-423. https://doi.org/10.1590/S0100-69162009000300008

Tat, M. E., \& Van Gerpen, J. H. (1999). The kinematic viscosity of biodiesel and its blends with diesel fuel. Journal of the American Oil Chemists' Society 76, 1511-1513. https://doi.org/10.1007/s11746-999-0194-0

Torrentes-Espinoza, G., Miranda, B. C., Vega-Baudrit, J., \& Mata-Segreda, J. F. (2017) Castor oil (Ricinus communis) supercritical methanolysis. Energy 140, 426-435. http://dx.doi.org/10.1016/j.energy.2017.08.122

Ventura, D. A. M. F., Alves, K. B., \& Santos, M. K. V. A. (2010). Análise comparativa entre o biodiesel de girassol e o biodiesel de mamona. In: IV Congresso Brasileiro de Mamona e I Simpósio Internacional de Oleaginosas Energéticas, João Pessoa, 2010.

Ventura, M., Deus, W. B., Silva, J. R., Andrade, L. H. C., Catunda, T., \& Lima, S. M. (2018). Determination of the biodiesel content in diesel/biodiesel blends by using the near-near-infrared thermal lens spectroscopy. Fuel 212, 309-314. https://doi.org/10.1016/j.fuel.2017.10.069

Vieira, B., Nadaleti, W. C., \& Sarto, E. (2021). The effect of the addition of castor oil to residual soybean oil to obtain biodiesel in Brazil: Energy matrix diversification. Renewable Energy 165, 657-667. https://doi.org/10.1016/j.renene.2020.10.056

Zuniga, A. D. G., Paula, M. M., Coimbra, J. S. R., Martins, E. C. A., Silva, D. X., \& TelisRomero, J. (2011). Revisão: Propriedades Físico-Químicas do Biodiesel. Pesticidas: Revista de Ecotoxicologia e Meio Ambiente 21, 55-72. http://dx.doi.org/10.5380/pes.v21i0.25939 